REVIEW

\title{
SYNCHRONOUS AND METACHRONOUS TUMORS - A LITERATURE REVIEW
}

\author{
Aurelia Nicoleta Sanda ${ }^{1,2}$, Roxana Ristea ${ }^{2}$, Ș. Neagu ${ }^{1,2,3}$ \\ ${ }^{1}$ Emergency University Hospital of Bucharest, Romania \\ ${ }^{2}$ Carol Davila University of Medicine and Pharmacy, Bucharest, Romania \\ ${ }^{3}$ Academy of Scientists from Romania
}

Corresponding author: Aurelia Nicoleta Sanda Email: nico.sanda@yahoo.com

\begin{abstract}
This paper aims to present the results of extensive studies on synchronous and metachronous tumors, both in order to optimize the surgical treatment of these, but also to concretize some suitable and useful screening measures for their early detection. The prognosis of cancer patients has improved over time. On the other hand, investigative methods have advanced considerably so that today are discovered tumors that could have gone unnoticed in the past. These data show why there are currently more cases of multiple cancers and their rate is steadily rising. The review will consider several studies focused on multiple tumors in terms of their localizations, manifestations, and risk factors. The first primary tumor found in a patient is called the index tumor, and the following tumors can be considered synchronous if they occurred in less than 6 months and metachronous if they occurred more than 6 months. Also, for the tumors of group IA, a variable of 4 centimeters was imposed13. Thus multiple tumors of the same tissue / organ (multicenter type A) are considered, tumors that are located at $4 \mathrm{~cm}$ or more of the index tumor, are histopathologically confirmed as malignant and are not metastasis or relapses of the first tumor.
\end{abstract}

KEYWORDS: multiple tumors, synchronous tumors, metachronous tumors

\section{INTRODUCTION}

The American Cancer Society estimates for 2018 the appearance of 1,735,350 new cases of cancer, of which colorectal cancers are approximately 150,000 . The number of deaths assessed for the United States this year was 609,640 , regardless of location and about 51,000 for colorectal cancers. (Cancer facts and figures, 2018) [1]. The study of multiple primary tumors can bring important information about the etiology and management of cancer in general.

In 1889, Theodor Bilroth (26 April 1829 - 6 Feb 1894) published the first case of multiple tumors (squamous cell carcinoma of the right ear and gastric carcinoma) and defined them as "two or more different tumors in one patient" [2]. In 1932 Warren S. and Gates O. made the first classification of multiple malignant tumors [3].

Colorectal cancer is one of the most common malignant tumors in the population, ranked third in both women (after breast and lung cancer), where it is estimated at over $9 \%$ of the US population in 2018 (75,610 new cases) and in men (after prostate and lung cancer), where it is estimated at about $7 \%$ of the US population in $2018(64,640$ new cases) [1]. Its association with other cancers is still an insufficiently studied topic. 
The prognosis of cancer patients has improved over time. On the other hand, investigative methods have advanced considerably so that today are discovered tumors that could have gone unnoticed in the past. These data show why there are currently more cases of multiple cancers and their rate is steadily rising.

Also, the average life span of the population has increased in the last decades, and the Earth's population currently has an average age of 28.4 years (27.7 years for men and 29 years for women in 2009) with a minimum of 15 years in the Niger population and a maximum of 40 years in the population of several European countries, Canada and Japan. According to a Global Social Change Research Project report, as the proportion of children (014 years) has decreased, the proportion of the elderly population (over 60 years) has increased, with an increase of $3 \%$ over the last 60 years worldwide $(8 \%$ of the total population of the Earth in 1950 and $11 \%$ in 2010) 4 and $10 \%$ at the level of Europe where in 1950 there were about 66 million people aged over 60 (12\%), and in 2010 their number grew to more than 161 million (22\%) [4]. In Romania of 2008, life expectancy at birth was 68.69 years for men and 75.89 years for women [5], and our country is in the group of states with negative spike in demography and has $14.7 \%$ of the population aged over 65 years [6]. All these demographic data aim to highlight a clear reality, namely that the increase in cancer incidence in the general population is obviously associated both with the increasing average life expectancy of the population and with the percentage increase of the elderly population phenomenon which is occurring in our country [6]. It is estimated that the rate of emergence of a new tumor in a patient already treated by the first is still underestimated due to the still high mortality of cancer patients $[7,8]$. Given that both in our country and in the world the life expectancy and the average age of the population are estimated to be increasing over the next decades5, we believe that the in-depth study of multiple cancers is of great importance.

\section{MATERIALS AND METHODS}

The research was based on the analysis of the papers from ScienceDirect, PubMed and WebofScience. The search used the formula: ("synchronous" OR "metachronous") AND "tumors", filtered by language (English).

\section{RESULTS AND DISCUSSIONS}

In 1930, Warren and Gates, studying 1259 patients with such pathologies, first defined multiple tumors as follows: (a) two histologically confirmed tumors as malignancies, (b) each of the cancers must be separate and distinct, (c) the second tumor must not be metastasis or relapse of the first tumor [3]. These criteria are currently preserved, and the distinction between synchronous and metachrone tumors is made by a time variable over which the majority of international oncology committees have agreed in the past 10 years to be 6 months $[8,9,10,11]$.

In 1964, Moertel proposed a classification, still used today for multiple malignant primary tumors [12].

\section{Multiple primary malignant tumors of multicentric origin}

\begin{tabular}{l}
$\begin{array}{l}\text { A. The same tissue and organ } \\
\text { (e.g. multiple } \\
\text { malignancies) }\end{array}$ \\
\hline B. A common tissue divided by \\
different organs (e.g. squamous \\
cell carcinoma of the larynx and \\
pharynx) \\
C. The same tissue in different \\
paired organs (e.g. bilateral breast \\
cancer)
\end{tabular}

II. Multiple primary malignant tumors originating in different tissues or organs (e.g. breast cancer and colorectal cancer)

III. Multiple primary malignant tumors plus a malignant lesion in another tissue or organ (e.g., 2 primary colon cancer and a third primary tumor in the cervix)

\section{Table 1 - Moertel Classification [12].}

The first primary tumor found in a patient is called the index tumor, and the following tumors can be considered synchronous if they occurred in less than 6 
months and metachronous if they occurred in more than 6 months $[8,9,10,11]$. Also, for the tumors of group IA, a variable of 4 centimeters was imposed [13]. Thus multiple tumors of the same tissue / organ (multicenter type A) are considered, tumors that are located at $4 \mathrm{~cm}$ or more of the index tumor, are histopathologically confirmed as malignant and are not metastasis or relapses of the first tumor [12].

Among the risk factors for colorectal cancer in general and for multiple tumors, in particular, a number of risk factors have been incriminated, among which: blood group A that appears to be associated with less aggressive tumor histology, age which also significantly influences histological grading, metabolic syndrome, eating habits, smoking, drinking alcohol and coffee [14].

A 23-year study in Poland, completed in 2014, evaluated 862 patients (570 men and 292 women) with gastric cancer. 58 of them (6.7\%) developed 62 multiple primary tumors: 39 metachrone $(63 \%)$ and 23 synchrone $(37 \%)$ [15]. The authors of this study argue that the most likely cause of a second primary cancers is the environmental factor as the most common malignancy following the gastric index tumor, was colorectal cancer (17 tumors). The second site of multiple primary tumors was in the order of frequency: lung ( 9 tumors), breast ( 8 tumors) and prostate (7 tumors). Thus, in a country where colorectal cancer, ranked fourth or fifth in the general population [5], is ranked first in patients with gastric index tumor. Esophageal cancer is not among the first places of multiple cancers after the gastric index tumor [15], which draws attention to the average factor and dietary habits that can affect the gastrointestinal tract.

Another important element highlighted by this study is the blood group of patients who develop a second primary malignant tumor after gastric cancer, thus recording a statistically significant difference in patients with blood group $0[14,15]$. Another statistically significant difference was the age of the patients. This study proves that they are more exposed to developing multiple malignant tumors, patients whose gastric index tumor appeared at a higher age [15]. In addition, patients diagnosed with synchronous tumors were older in age than those diagnosed with metachronous tumors $[14,15]$.
Another large-scale malignant tumor study was published in 2012 and was conducted over a period of 20 years (1985-2004) in Osaka, Japan. The study starts from the premise that in Japan the risk of cancer is $50 \%$ for men and $40 \%$ for women, and at least half of these patients will live for at least 5 years after the first cancer, and in this case cancer survivors are at risk of making a second primary cancer. Unlike other studies, it changed the time variable so that they considered multiple malignancies only tumors that occurred after 3 months and less than 10 years of the index tumor; also, the index tumor did not have a specified location, it could belong to any system or organ. Multiple malignant tumors defined as such occurred in 13,385 patients of 355,966 cancer survivors, which is $3.8 \%$ in the first 10 years. The risk of developing a new primary tumor was much higher in the 60-69 age group $13 \%$ ( $16.2 \%$ for males and $8.6 \%$ for females). The authors highlight the twice bigger proportion of multiple cancers over the past 20 years, observation which is based on another study on the same subject conducted by the same team between 1966 and 1986. A possible explanation for this may be the accumulation of risk factors, but a significant improvement in diagnostic measures should not be omitted.

A great value in this study is the individualization of index and successive cancers according to localization. Thus, the most common 3 localization of malignant index tumors were in the pharynx, esophagus and stomach. Successive malignant tumors were found in this study according to the following table [16].

\begin{tabular}{lll}
\hline Pharynx & 1. & Esophagus \\
& 2. & Larynx \\
& 3. & Lung \\
\hline Esophagus & 1. & Pharynx \\
& 2. & Larynx \\
& 3. & Pancreas and \\
& \multicolumn{3}{l}{ urinary system } & Thyroid \\
Stomach & 2. & Esophagus \\
& 3. & Pharynx \\
\hline
\end{tabular}

Table 2 - Localization of successive different tumors [16]. 
The most important association between index and successive tumor localizations was for the breast, uterus and ovary, especially when it comes to breast tumor index. Equally, localizations in the pharynx, esophagus and larynx have a high association and statistically evident association. Additionally, thyroid malignancies appear to occur with high frequency as successive tumors, but not as index tumors [16].

The third study was also conducted in Japan using Nagasaki prefecture cancer registry data; it was published in 2014 and was conducted over a period of 23 years (19852007), involving 174,477 patients with an index tumor, of which $14,167(8.1 \%)$ were diagnosed with a primary successive malignancy. This study clearly shows that the risk of being diagnosed with a malignant tumor is significantly higher in patients previously diagnosed with cancer than in the general population and raises the issue of the need to standardize the oncological follow-up of these patients [17]. The authors considered that for the optimization of follow-up the most important is to determine a certain association of the localization of cancers in order to focus on targeted investigations for certain systems and organs. Therefore, for the initial pharyngeal or oral cavity cancer, the second most frequent localization was in the esophagus, while for the location of the tumor in the esophagus, the most frequent localization of the second primary malignant tumor was in the stomach, and gastric index tumors are most frequently followed by colorectal malignant tumors.

In this study, successive tumors occurred frequently in the first year of tumor diagnosis, decreased as frequency in the second year after first diagnosis, followed by a steady increase in the frequency in the next 10 years after diagnosis, when their number was high again. There was a significant decrease in the occurrence of successive tumors 20 years after the diagnosis of the tumor [17].

It has also been investigated the involvement of certain risk factors concentrated on geographical areas and cultural regions. Comparing this study with another one conducted in Australia between 1997 and 2001, in which successive tumors do not affect the esophagus and ovary to the same extent, being more common in the head and neck [18], it was concluded that genetic factors, smoking and alcohol consumption are certain determinants of the localization of multiple malignant tumor [17]. Another explanation of the differences between studies may be the time and methods of tracking patients with different index tumors.

In 2015, Takahiro Tabuchi and colleagues, published in the International Journal of Cancer, a study of risk factors that predispose to the occurrence of a successive malignant tumor, primarily considering smoking and drinking alcohol. They compared 27,762 patients with a malignant tumor diagnosed between 1985 and 2007. The risk of developing a second primary cancer is, according to this study, of $43-108 \%$ of patients with occasional tobacco / alcohol consuming, 51-126\% of those who drink / smoke currently and 167-299\% of patients with high consumption of alcoholic beverages / tobacco [16,20]. Of the total survivors of a first cancer, $27 \%$ said they did not ever drink or smoke and $44.9 \%$ said they were drinking or smoking occasionally [20]. During monitoring, $6.4 \%$ of patients were diagnosed with a second primary malignancy, $0.4 \%$ with the third and $0.04 \%$ with the fourth [20].

The risks of drinking alcohol and smoking have been assessed separately. It was found that the successive tumors risk of patients with high alcohol consumption prior to the first diagnosis is significantly higher than those who did not previously drink alcohol [21]. On the contrary, occasional alcohol consumers have no greater risk, whether they have discontinued their consumption or not. Similarly, large smokers have been identified as having a much higher risk and even a smoking dose-related risk has been identified. However, unlike alcohol, smoking even occasionally or at low dose, increases the risk of a successive malignant tumor [20,22].

Reported to the location of successive tumors, the authors found that regardless of the location of the index malignant tumor, in patients without previous ethanol or tobacco use who developed a second primary malignancy, it was at the level of breast $[20,23]$, urinary tract $[20,24]$, thyroid or was of hematological type $[20,25]$ while in smokers and drinkers the risk was higher for the following locations: oral cavity, pharynx, esophagus, stomach, colon and 
rectum, liver, larynx, lung, prostate and urinary tract [25]. Casual alcohol or tobacco consumers had statistically significantly higher risk for successive malignant tumors of the esophagus and lung while patients with average alcohol / tobacco consumption had a higher risk of successive tumors in the esophagus, the stomach or the lung. The risk of high-smoking or alcohol-consuming patients of developing a new malignant primary tumor involved in particular the oral cavity and pharynx, and less the esophagus, stomach and lung.

An interesting aspect of this study is that patients with occasional alcohol or tobacco consumption have shown a lower risk than patients who have never consumed alcohol or tobacco. However, the authors do not recommend that these substances be consumed in moderate amounts after the first diagnosis of malignancy, as these two substances are however defined as carcinogens by the International Agency for Research on Cancer. The study concludes that to reduce the risk of developing primary malignant tumors in patients with cancer should: (i) reduce or stop drinking alcohol, (ii) completely stop smoking and (iii) patients with both tobacco and alcohol use should be considered as having a very high risk and should be treated with adequate riskmanagement advice [20].

In a SEER (Surveillance, Epidemiology and End Results) analysis of the 1973-1999 National Cancer Institute of the United States of America conducted on over 2.7 million cases of cancer, $10 \%$ of them were diagnosed with a successive primary malignant tumor, and $2-12 \%$ of those who already had 2 primary tumors tend to develop a third primary malignant tumor [26]. The risk factors inherited in this analysis are (a) the index tumor itself, (b) the location of the first tumor - a higher preponderance of successive tumors has been revealed when the index tumor is located at the head and neck, (c) persistent carcinogenic factors after the treatment of the first tumor, (d) genetic predisposition, (e) oncological therapy whether it is radiotherapy, chemotherapy or genetic manipulation, and (f) immunosuppression [16,26].

\section{CONCLUSIONS}

All of these studies raise multiple questions but also establish the need for a continuous study of multiple cancers, as by dissecting them we will get to deepen the origin and the way of development of malignant neoplasms, the full understanding of cancer being the only way by which the targeted treatment, and prophylaxis of the population at risk, should lead in the near future to the eradication of most of the malignant tumors. The association of certain cancers, localizations and risk factors are important elements that can be studied in detail in patients known to already have an important risk factor, namely malignant tumor index.

\section{REFERENCES}

[1] Facts and Figures 2018, cancer.org/statistics and Cancer Statistics 2018, cancerjournal.com

[2] Kazi RA. , Peter RE. Christian Albert Theodor Bilroth: Master of surgery, Journal of postgraduate Medicine 2004, Vol. 50, PG 82-83

[3] Warren S., Gates O. Multiple malignant tumors: a survey of the literature and statistical study Am J Cancer 1932; Vol. 16: pg 1358-1414

[4] Shakman, gene, Xun Wang and Ya-Lin Liu 2012. Brief Review of world demographic trendstrends in age distribution. The Global social Change Research Project. Available at http://gsociology.icaap.org/report/demsumAging.ht $\mathrm{ml}$

[5] World Health Organization "Life expectancy" http://apps.who.int/gho/data/node.main.688

[6] Institutul National de Statistica Bucuresti, Recensamantul populatiei locuintelor 2011 Bucuresti, Iulie 2013

[7] Neugut AI, Robinson E. Multiple primary neoplasms. Cancer J 1992; vol 5; pg 245-248.

[8] Luciani A. , Ascione G. , Marussi D. , Caldiera S. , Bozzoni S. , Codeca C. , Zonato S. Ferrari D. , Foa P. Clinical analysis of multiple primary malignancies in the eldery. Med. Oncol 2009; vol 26; pg 27-31

[9] Frodin JE, Ericsson J., Barlow L. Multiple primary malignant tumors in national cancer registry - reliability of reporting. Acta Oncol.1997; Vol 36; pg 465-469.

[10] Cunliffe WJ, Hasienton PS, Tweddie DE, Schofield PF. Incidence of synchronous and metachronous colorectal carcinoma. Br J Surg 1984; Vol 71,pg 941-943 
[11] Moertel GG, Bargen JA, Dockerty MB. Multiple carcinomas of large intestine; A review of the literature and study of 261 cases, Gastroenterology 1958 Vol 34 pg 85-98

[12] Charles G. Moertel, Multiple primary malignant neoplasms- historical perspectives, cancer 40: 17861792.Doi:10.1002/1097-0142(197710)40:4+<1786:: AID-CNCR2820400803>3.0.CO;2-2

[13] N Tziris, J Dokmetzioglou, K Giannoulis, I Kesisoglou, K Sapalidis, E Kotidis, O Gambros. Synchronous and metachronous adenocarcinomas of large intestine. Hippokratia. 2008; Jul-Sep; 12(3): $150-152$

[14] Meda-Laura Comandasu, Neagu Stefan-Ilie, Leziuni neoplazice sincrone in cancerele colorectale, 2016, pg 143-152

[15] Malgorzata Ławniczak, Alicjia Gawin, Halina Jaroszewicz-Heigelmann, Wielslawa RogozaMateja, Joanna Raszeja Wyszomirska, Andrzej Bialek, Katarzyna Karpinska -Kaczmarczyk and Teresa Starzynska. Synchronous and Metachronous neoplasm in gastric cancer patients: A 23 year study, World Journal of Gastroenterology, 21 jun 2014, vol $20 \quad$ (23); $\quad$ pg $\quad 7480-7487$ doi:10.3748/wjg.v20.i23.7480.

[16] Tabuchi, T. , Ito, Y. , Ioka, A. , Miyashiro, I. and Tsukuma, H. (2012), Incidence of metachronous second primary cancers in Osaka, Japan: Update of analyses using population-based cancer registry data. Cancer Sci, 103: 1111-1120. doi:10.1111/j.1349-7006.2012.02254.x

[17] Mai Utada, Yuko Ohno, Megumi Hori, Midori Soda, Incidence of multiple primary cancers and interval between first and second primary cancers, 11 may 2014, Csancer Sci (2014) 890-896. doi: https://doi.org/10.1111/cas.12433

[18] Youlden DR, Baade PD. The relative risk of second primary cancers in Queensland, Australia: a retrospective cohort study. BMC Cancer2011;11: 83.
[19] Curtis RE, Boice JD Jr, Kleinerman RA, Flannery JT, Fraumeni JF Jr. Sum-mary: multiple primary cancers in Connecticut, 1935-82 Natl Cancer Inst Monogr 1985; 68: 219-42.

[20] Tabuchi, T. , Ozaki, K. , Ioka, A. and Miyashiro, I. (2015), Joint and independent effect of alcohol and tobacco use on the risk of subsequent cancer incidence among cancer survivors: A cohort study using cancer registries. Int. J. Cancer, 137: 2114-2123. doi:10.1002/ijc. 29575

[21] Inoue $M$, Nagata $C$, Tsuji I, et al. Imp act of alcohol intake on total mortality and mortality from major causes in japan: a pooled analysis of six largescale cohort studies. J Epidemiol Communit y Health 2012;66:448-56.

[22] Ide R, Mizoue T, Fujino Y, et al. Cigarette smoking, alcohol drinking, and oral and pharyngeal cancer mortality in japan. Oral Dis 2008;14:314-9.

[23] Knight JA, Bernstein L, Largent $J$, et al. Alcohol intake and cigarette smoking and risk of a contralateral breast cancer: the women's environmental cancer and radiation epidemiology Study. Am J Epidemiol 2009;169:962-8.

[24] Nasca P. Tobacco and cancer. In: Nasca P, Pastides H, eds. Fundamentals of cancer epidemiology, 2nd edn. Canada: Jones and Bartlett Publishers, Inc., 2008. 178-224.

[25] Park SM, Lim MK, Jung KW, et al. Prediagnosis smoking, obesity, insulin resistance, and second primary cancer risk in male cancer survivors: national health insurance corporation Study. J Clin Oncol 2007;25:4835-43.

[26] Howlader N, Noone AM, Krapcho M, Miller D, Bishop K, Kosary CL, Yu M, Ruhl J, Tatalovich Z, Mariotto A, Lewis DR, Chen HS, Feuer EJ, Cronin KA (eds). SEER Cancer Statistics Review, 19752014, National Cancer Institute. Bethesda, MD, https://seer.cancer.gov/csr/1975_2014/, based on November 2016 SEER data submission, posted to the SEER web site, April 2017. 